\title{
FT-IR studies of sickle hemoglobin interaction with phosphatidylserine
}

\author{
Hao Ouyang a,1, David J. Moore ${ }^{\mathrm{a}, 1, *}$, Richard H. Sills ${ }^{\mathrm{b}}$ and Richard Mendelsohn ${ }^{\mathrm{a}}$ \\ ${ }^{a}$ Department of Chemistry, Rutgers University, Warren Street, Newark, NJ 07102, USA \\ ${ }^{\mathrm{b}}$ Department of Pediatrics, Albany Medical College, New Scotland Road, Albany, NY, USA
}

\begin{abstract}
The interaction of dilauroylphosphatidylserine (DLPS) vesicles with both normal human hemoglobin (HbA) and hemoglobin from patients with sickle cell disease ( $\mathrm{HbS}$ ) has been investigated with FTIR spectroscopy. Changes in the conformational order of the phospholipid chains were directly monitored via the acyl chain $\mathrm{CH}_{2}$ symmetric stretching mode frequencies. The hemoglobin oxygenation state was simultaneously monitored via the $\mathrm{S}-\mathrm{H}$ stretching contour. The current study reveals that under oxygenated conditions, DLPS shows little interaction with either HbA or HbS. In contrast, deoxyHbS interacted with DLPS by abolishing the gel-liquid crystal phase transition.
\end{abstract}

Keywords: Erythrocytes, FTIR spectroscopy, hemoglobin, membrane, lipid-protein interactions, conformational order

\section{Introduction}

The asymmetric composition and distribution of lipid across the human erythrocyte membrane has been extensively documented $[3,4,14]$. The outer membrane leaflet is enriched in phosphatidylcholine (PC) and sphingomyelin (SM), whereas the inner layer consists primarily of phosphatidylethanolamine (PE) and phosphatidylserine (PS). Under normal physiologic conditions, the lipids of the erythrocyte membrane are in a liquid-crystalline state, in which the lipids are constantly undergoing intramolecular trans/gauche isomerization. In addition to any lateral movements within the bilayer plane, transmembrane proteins also continuously transport aminophospholipids (PS and PE) to the inner side of the membrane [5]. The significance of lipid asymmetry for the physiological functions of erythrocytes remains to be clearly established, although there is increasing evidence that lipid organizational heterogeneity plays an important role in regulating membrane protein activity and biochemical signaling [3-6].

Hemoglobin, which exists in the erythrocyte at a tetramer $\left(\alpha_{2} \beta_{2}\right)$ concentration of $\sim 5 \mathrm{mM}$, is the major oxygen carrier in blood. A variety of hemoglobin mutations have been identified, the most common among these causes sickle cell anemia. The difference between normal human hemoglobin (HbA) and sickle cell hemoglobin ( $\mathrm{HbS})$ is a single amino acid change from glutamic acid to valine in the $\beta$ polypeptide chain. This single amino acid change is associated with a change in protein conformation upon deoxygenation that allows for $\mathrm{HbS}$ polymerization. The formation of $\mathrm{HbS}$ polymers ultimately impacts upon erythrocyte morphology inducing a shape change from a biconcave disc to a sickle cell $[7,8]$.

Sickle cell membranes are similar to normal red blood cells under conditions of adequate oxygenation. However, $\mathrm{Hb}$ deoxygenation induces a redistribution of membrane phospholipids in sickle cells, but not

\footnotetext{
${ }^{1}$ Present address: Unilever Research US, River Road, Edgewater, NJ 07020, USA.

${ }^{*}$ Corresponding author. E-mail: david.j.moore@unilever.com.
} 
in normal cells. The outer leaflets of sickle cells become enriched in PS and PE while some outer leaflet PCs translate to the inner monolayer [6]. PS in the outer leaflet of the bilayer serves as a lipid source in the coagulation scheme, while PE on a cell surface is a target for macrophage recognition [7]. Deoxygenation of sickle hemoglobin distorts the erythrocyte membrane, destroying normal phospholipid asymmetry and thereby influencing sickle cell aggregation and recognition. A previous study [14] emphasized possible structural changes in hemoglobin during protein-lipid interaction. The effect of this interaction on the molecular organization and conformation of membrane lipids has not been directly illustrated.

This laboratory has a long-standing interest in the molecular dynamics of lipid organization in membranes, including those of human erythrocytes. In previous studies [10-12] we have developed novel FTIR spectroscopy techniques, and demonstrated that these are useful, non-perturbative methods for monitoring phospholipid organization and conformational order of particular lipid species in erythrocyte membranes. The current work describes an FTIR spectroscopic technique based upon the previous pioneering studies of Alben \& colleagues [1,9] on $\mathrm{Hb}$ solutions which allows direct determination of $\mathrm{Hb}$ oxygenation in intact erythrocytes. The ability to monitor thermotropic changes in $\mathrm{HbA}$ and $\mathrm{HbS}$ structure as a function of oxygenation is demonstrated, as are changes in lipid conformational order during the interaction of model phospholipids with oxygenated and deoxygenated $\mathrm{HbS}$ and $\mathrm{HbA}$.

\section{Materials and methods}

\subsection{Materials}

Dilauroylphosphatidylserine (DLPS) was purchased from Avanti Polar Lipids (Alabaster, AL). All other chemicals were of cell culture grade and purchased from Sigma Chemical Co. (St. Louis, MO).

\subsection{Hemoglobin solutions}

Fresh human blood samples from healthy adults and patients with sickle cell anemia were collected by venipuncture into heparin tubes following protocols approved by the Institutional Review Board. The isolation of hemoglobin followed standard procedures and is outlined below. After removing the buffy coat by aspiration of centrifuged blood, erythrocytes were washed three times with $1 \%$ saline and hemolyzed with 1 volume of $\mathrm{H}_{2} \mathrm{O}$ (HPLC grade) and 0.5 volume toluene. After centrifugation hemolysates were passed through a Sephadex G-25 column and equilibrated with phosphate buffered saline (PBS): $138 \mathrm{mM} \mathrm{NaCl}, 5 \mathrm{mM} \mathrm{KCl}, 6.1 \mathrm{mM} \mathrm{Na} 2 \mathrm{HPO}_{4}, 1.4 \mathrm{mM} \mathrm{NaH} \mathrm{PO}_{4}, 0.01 \mathrm{mM}$ EGTA, $\mathrm{pH}=7.3$. The resulting solutions were concentrated by Amicon filtration (Amico, Beverly, MA) to a final heme concentration of 18-21 mM, giving a hemoglobin tetramer concentration of $\sim 5 \mathrm{mM}$ which is equivalent to normal intracellular concentration. The cutoff molecular weight of the filtration was 10,000 and the final protein concentration was monitored by UV-Vis spectrophotometry at $575 \mathrm{~nm}$ for oxyhemoglobin.

\subsection{Sample preparation}

Five milligrams of lipid powder was dissolved in chloroform, then dried under a stream of $\mathrm{N}_{2}$ gas followed by pumping under vacuum. Vesicles containing oxygenated hemoglobin were prepared by adding $50 \mu \mathrm{l}$ of $\mathrm{Hb}$ solution to the dried lipid film and mixing the sample by vortex action at $25^{\circ} \mathrm{C}$ (i.e., above 
the gel-liquid crystal phase transition temperature of DLPS). To prepare deoxyhemoglobin containing vesicles, sodium dithionite was dissolved in the hemoglobin solution prior to addition of $50 \mu$ lo the dried lipid. The sodium dithionite protocol generates irreversibly deoxygenated hemoglobin [8]. Conversion of oxyhemoglobin to deoxyhemoglobin was monitored in UV-VIS spectra via the disappearance of the double peaks at $\sim 545$ and $575 \mathrm{~nm}$ and the appearance of the characteristic peak of deoxyhemoglobin at $\sim 555 \mathrm{~nm}$. Oxygenated and deoxygenated erythrocyte samples were prepared by washing cells in phosphate buffered saline (PBS) or PBS with sodium dithionite, respectively, then concentrated by gentle centrifugation.

\subsection{Methods for FTIR spectroscopy and data processing}

Both lipid-hemoglobin and erythrocyte samples were sandwiched between $\mathrm{CaF}_{2}$ windows and mounted in a temperature-controlled transmission cell (Harrick Scientific, Ossining, NY). Temperature was controlled with a circulating water bath (Fisher Scientific, Springfield, NJ). Spectra were acquired over a range of $20^{\circ} \mathrm{C}$ at $1-2^{\circ} \mathrm{C}$ intervals. All spectra were acquired on a Mattson RS- 1 spectrometer by co-addition of 512-interferograms collected from 4000 to $400 \mathrm{~cm}^{-1}$ at $4 \mathrm{~cm}^{-1}$ resolution under continuous $\mathrm{N}_{2}$ purge. These were apodized with a triangular function and Fourier transformed with one level of zero filling. Second derivative spectra were generated and peak positions were determined using a center of gravity algorithm.

In the current study we used FTIR spectroscopy to directly measure the S-H stretching modes of specific cysteine residues in the hemoglobin tetramer in both oxygenated and deoxygenated states. Our data are in excellent agreement with the studies of Alben and colleagues [1,9] in which detailed assignments of hemoglobin cysteine S-H modes were deduced from animal and human hemoglobin. The conformational order of lipid acyl chains were also measured by FTIR spectroscopy by monitoring the $\mathrm{CH}_{2}$ symmetric stretching mode frequencies [10-12].

\section{Results}

Figures 1A and 1B display the original and inverted second derivative spectra in the $2535-2580 \mathrm{~cm}^{-1}$ spectral region of oxygenated and deoxygenated sickle hemoglobin within intact human erythrocytes from a sickle cell anemia patient. The modes in this spectral region arise from cysteine $\mathrm{S}-\mathrm{H}$ stretching vibrations in human hemoglobin, and have been assigned by Alben and co-workers as the $\alpha 104$ cysteine S-H stretching mode $\left(\sim 2555 \mathrm{~cm}^{-1}\right)$ and the $\beta 112$ cysteine $\mathrm{S}-\mathrm{H}$ stretching mode $\left(2565 \mathrm{~cm}^{-1}\right)$ [1,9]. In the oxygenated intact erythrocytes the $\alpha 104$ and $\beta 112$ cysteine $\mathrm{S}-\mathrm{H}$ modes appear at 2553 and $2567 \mathrm{~cm}^{-1}$, respectively, whereas in the deoxygenated cells the $\alpha 104$ cysteine $\mathrm{S}-\mathrm{H}$ shifts to $2557 \mathrm{~cm}^{-1}$ and the $\beta 112 \mathrm{~S}-\mathrm{H}$ mode is no longer visible. These frequency shifts result from changes in the hydrogen bonding of the cysteine $\mathrm{S}-\mathrm{H}$ groups, which are a consequence of changes in the ternary and quaternary structure of the hemoglobin tetramer induced by deoxygenation. In Fig. 1C, the inverted second derivative spectra in the same spectral region for oxygenated and deoxygenated isolated sickle cell hemoglobin in the presence of DLPS are shown for comparison. The similarity between Figs 1B and 1C demonstrates that the oxygenated states of $\mathrm{HbS}$ could be monitored when it interacts with lipid species.

The temperature dependence of the $\alpha 104$ cysteine S-H mode is plotted in Fig. 2A for oxygenated and deoxygenated intact sickle cell erythrocytes. In the oxygenated cells there is a progressive increase in the S-H stretching frequency from 2553.5 to $2556 \mathrm{~cm}^{-1}$ over the temperature range 25 to $55^{\circ} \mathrm{C}$. In contrast, the deoxygenated cells show no temperature dependence in the $\alpha 104$ cysteine S-H mode frequency, 


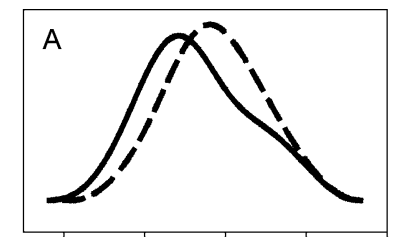

25402550256025702580
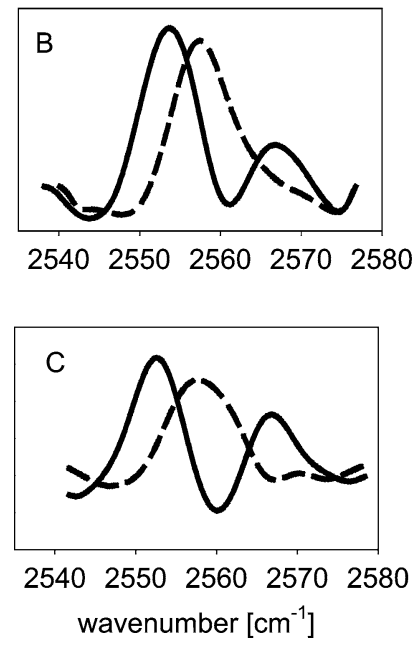

Fig. 1. (A): Original spectra of S-H stretching modes of oxygenated (solid line) and deoxygenated (dash line) HbS in the 2540-2580 $\mathrm{cm}^{-1}$ spectral region. These spectra were obtained from intact red blood cell. (B): Inverted second derivative spectra of S-H stretching modes of oxygenated (solid line) and deoxygenated (dash line) HbS of intact red blood cell in the $2540-2580 \mathrm{~cm}^{-1}$ spectral region. (C): Inverted second derivative spectra of S-H stretching modes of oxygenated (solid line) and deoxygenated (dash line) HbS hemoglobin isolated from the red blood cell. These spectra were obtained in the presence of DLPS.

which remains constant at $\sim 2556 \mathrm{~cm}^{-1}$ over the studied temperature range. In Fig. $2 \mathrm{~B}$, the temperature dependence of the $\alpha 104$ cysteine S-H mode frequency was measured in the presence of DLPS for both oxygenated and deoxygenated $\mathrm{HbS}$. Since it is desirable to be able to monitor the conformational order of the lipid and the oxygenation state of the protein simultaneously, the temperature range for this model system was adjusted to $8-32^{\circ} \mathrm{C}$ ( $T_{\mathrm{m}}$ for pure DLPS is about $18^{\circ} \mathrm{C}$ ). The thermotropic behaviors of both oxygenated and deoxygenated hemoglobin in this simple protein-lipid interaction model are similar to those in the intact sickle cell.

Figure $3 \mathrm{~A}$ shows the temperature dependence of the conformationally sensitive $\mathrm{CH}_{2}$ symmetric stretching mode frequency $\left(\nu_{\mathrm{s}} \mathrm{CH}_{2}\right)$ of DLPS in the presence of oxygenated $\mathrm{HbA}$ (normal adult hemoglobin) and $\mathrm{HbS}$ (sickle cell hemoglobin). In both samples the transition is sharp, cooperative, and occurs at the temperature of pure DLPS. This indicates that no significant interaction is occurring between oxygenated hemoglobin in solution (both $\mathrm{HbA}$ and $\mathrm{HbS}$ ) and the DLPS membranes. The equivalent parameters are plotted for deoxygenated hemoglobin samples in Fig. 3B. The thermotropic response of $\nu_{\mathrm{s}} \mathrm{CH}_{2}$ from DLPS vesicles containing deoxygenated $\mathrm{HbA}$ indicates some interaction between deoxygenated $\mathrm{HbA}$ and DLPS. The initial and final frequencies, 2848.8 and $2850.5 \mathrm{~cm}^{-1}$, respectively, are more ordered than those measured from oxygenated samples. Furthermore, while $T_{\mathrm{m}}$ remains approximately constant at $\sim 18^{\circ} \mathrm{C}$, the transition is broadened, further indicating some interaction between the deoxygenated $\mathrm{HbA}$ and DLPS. The thermotropic response of $\nu_{\mathrm{s}} \mathrm{CH}_{2}$ from DLPS vesicles contain- 

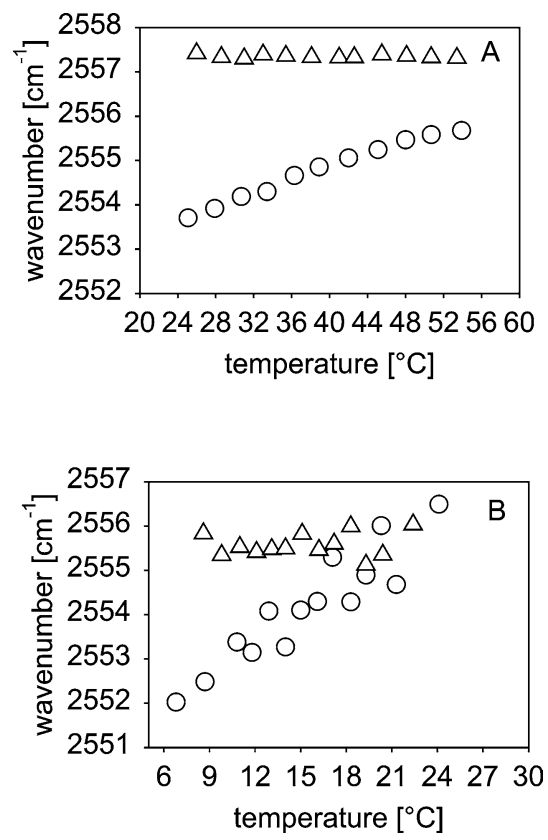

Fig. 2. (A) The thermotropic behaviors of S-H stretching mode ( $\alpha 104$ cysteine) of oxygenated (circle) and deoxygenated (triangle) HbS of intact red blood cell. (B) The thermotropic behaviors of S-H stretching mode ( $\alpha 104$ cysteine) of oxygenated (circle) and deoxygenated (triangle) HbS isolated from the red blood cell. These spectra were obtained in the presence of DLPS.
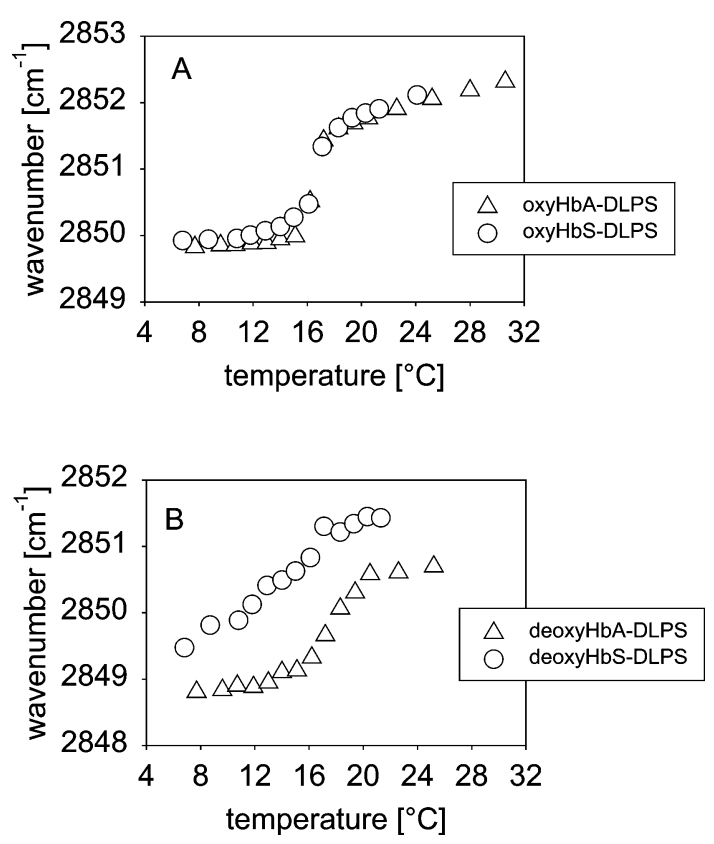

Fig. 3. The thermotropic responses of the symmetric $\mathrm{CH}_{2}$ stretching mode of DLPS incorporated with normal hemoglobin (triangle) and sickle hemoglobin (circle) under both oxygenated (A) and deoxygenated (B) conditions. 
ing deoxygenated $\mathrm{HbS}$ shows a significant change from the oxygenated sample. The frequency range suggests more order than the corresponding oxygenated sample $\left(2849.5-2851.5 \mathrm{~cm}^{-1}\right.$ versus 2849.9 $2852 \mathrm{~cm}^{-1}$ ), however, there is no indication of a cooperative transition but rather a monotonic increase in frequency as temperature is increased. This suggests a significant interaction of deoxygenated $\mathrm{HbS}$ with DLPS membranes.

\section{Discussion}

Since lipid behavior and protein structure can be simultaneously monitored in a single IR spectrum, biophysical FTIR spectroscopy methods are well suited to studying intermolecular and intramolecular lipid organization in membranes, as well as specific lipid-protein interactions. In the last decade our laboratory has successfully developed experimental techniques to probe lipid organization and conformational order in the membranes of intact normal human erythrocytes [10-12]. The current study describes initial experiments aimed at expanding this approach to sickle cell anemia erythrocytes. To that end the current FTIR experiments exploit changes in the $\alpha 104 \mathrm{~S}-\mathrm{H}$ stretching mode frequency, originally assigned by Alben and colleagues [1,9], to directly monitor hemoglobin oxygenation (UVVis spectrometry measurements were also taken as controls in these initial experiments). In this initial study, we have demonstrated that we are able to monitor the $\nu \mathrm{S}-\mathrm{H}$ mode of $\alpha 104$ cysteine within intact erythrocytes.

In future work the above approach will be utilized to directly monitor $\mathrm{Hb}$ oxygenation while simultaneously measuring the conformational order of specific lipid classes in erythrocytes. However, as a precursor to such studies, we have examined $\mathrm{Hb}$-lipid interactions in single component phospholipid vesicles with normal and sickle human hemoglobin ( $\mathrm{HbA}$ and $\mathrm{HbS}$ ). Although a simplified model membrane system, this experimental approach permits detailed and unambiguous examination of the molecular interactions between hemoglobin and a specific phospholipid class, i.e., phosphatidylserine. Utilizing single component vesicles provides some insight into the consequence of phospholipid asymmetry in erythrocyte membranes. Thus, for example, the extent of interaction between oxygenated or deoxygenated $\mathrm{Hb}$, and PS, can be compared for normal and sickle hemoglobin without the complications that might arise from the additional compositional complexity in native membranes. Clearly, if no interactions were observed in the model system it is unlikely that we would detect anything in the intact cells.

The methylene stretching modes $\left(\nu_{\mathrm{s}} \mathrm{CH}_{2}\right.$ and $\left.\nu_{\mathrm{as}} \mathrm{CH}_{2}\right)$ of lipid chains are sensitive to chain conformational order such that when lipid chains disorder, through the introduction of gauche rotamers, both $\nu_{\mathrm{s}} \mathrm{CH}_{2}$ and $\nu_{\mathrm{as}} \mathrm{CH}_{2}$ increase. This is clearly seen for our control experiments (Fig. 3) and numerous previous studies (for a thorough review, see [2]). The data for the PS-Hb model clearly reveal a strong $\mathrm{Hb}$-lipid interaction in the case of the deoxygenated $\mathrm{HbS}$, but not for the oxygenated HbS-PS system. In contrast, the PS chains in the HbA sample do not show evidence of a strong lipid-protein interaction, regardless of $\mathrm{Hb}$ oxygenation. In normal erythrocytes, PS is located entirely on the internal leaflet of the membrane bilayer. The significant impact of deoxygenated HbS upon PS membrane lipid organization is directly observed in these experiments via the abolition of the lipid phase transition and the fluidizing of chains, even at temperatures some $10^{\circ} \mathrm{C}$ below $T_{\mathrm{m}}$ of the pure lipid. The FTIR experimental approach permits the direct monitoring of lipid chain conformational order, not changes in probe or label properties, thus the current data clearly show a strong interaction between PS lipids and deoxygenated HbS. The lack of perturbation in the phase behavior of PS with $\mathrm{HbA}$ indicates that deoxygenation does not result in any significant disruption of PS chains. 
The interaction observed here, between deoxygenated $\mathrm{HbS}$ and PS, may well be of physiological relevance. We have previously developed an experimental method that permitted the measurement of PS conformational order in the inner and outer leaflet of intact erythrocytes as a function of cell shape. A synthesis of the current methods (direct $\mathrm{Hb}$ monitoring in intact cells) and findings (deoxygenated $\mathrm{HbS}-\mathrm{PS}$ interactions) and our previous approach will permit us probe the extent of changes in cell membrane lipid organization, as a function of lipid class and $\mathrm{Hb}$ state, within intact cells.

\section{References}

[1] G.H. Bare, J.O. Alben and P.A. Bromberg, Sulfhydryl groups in hemoglobin. A new molecular probe at the $\alpha_{1} \beta_{1}$ interface studied by Fourier Transformed Infrared Spectroscopy, Biochemistry 14 (1975), 1578-1583.

[2] G. Cevc and D. Marsh, Phospholipid Bilayers, John Wiley \& Sons, New York, 1987.

[3] D. Chiu, B. Lubin, B. Roelofsen and L.M. van Deenen, Sickle erythrocytes accelerate clotting in vitro: an effect of abnormal membrane lipid asymmetry, Blood 58 (1981), 398-401. E. Marva and R.P. Hebbel, Denaturing interaction between sickle hemoglobin and phosphatidylserine liposomes, Blood 83 (1994), 242-249.

[4] P.F. Devaux, Static and dynamic lipid asymmetry in cell membranes, Biochemistry 30 (1991), 1163-1173.

[5] P.F.H. Franck, D. Chiu, J.A.F. Op den Kamp, B. Lubin, L.M. van Deenen and B. Roelofsen, Accelerated transbilayer movement of phosphatidylcholine in sickled erythrocytes, J. Biolog. Chem. 258 (1983), 8435-8442.

[6] F.H. Franck, E.M. Bevers, B.H. Lubin, P. Comfurius, D. Chiu, J.A.F. Op den Kamp, R.F.A. Zwaal, L.L.M. van Deenen and B. Roelofsen, Uncoupling of the membrane skeleton from the lipid bilayer, J. Clin. Investig. 75 (1985), 183-190.

[7] R.P. Hebbel, Beyond hemoglobin polymerization: the red blood cell membrane and sickle disease pathophysiology, Blood 77 (1991), 214-237.

[8] J. Hofrichter, P.D. Ross and W.A. Eaton, Kinetics and mechanism of deoxyhemoglobin S gelation: a new approach to understanding sickle cell disease, Proc. Nat. Acad. Sci. (USA) 17 (1974), 4864-4868.

[9] P.P. Moh, F.G. Fiamingo and J.O. Alben, Conformational sensitivity of $\beta-93$ cysteine SH to ligation of hemoglobin observed by FT-IR spectroscopy, Biochemistry 26 (1987), 6243-6249.

[10] D.J. Moore, R.H. Sills, N. Patel and R. Mendelsohn, Conformational order of phospholipids incorporated into human erythrocytes: an FTIR spectroscopy study, Biochemistry 35 (1996), 229-235.

[11] D.J. Moore, R.H. Sills and R. Mendelsohn, Conformational order of specific phospholipids in human erthrocytes: correlations with changes in cell shape, Biochemistry 36 (1997), 660-664.

[12] D.J. Moore, S. Gioioso, R.H. Sills and R. Mendelsohn, Some relationships between membrane phospholipid domains, conformational order, and cell shape in intact human erythrocytes, Biochim. Biophys. Acta 1415 (1999), 342-348.

[13] Y. Shiviro, I. Zilber and N. Shaklai, The interaction of hemoglobin with phosphatidylserine vesicles, Biochim. Biophys. Acta 687 (1982), 63-70.

[14] R.F.A. Zwaal and A.J. Schrodit, Pathophysiologic implications of membrane phospholipid asymmetry in blood cells, Blood 89 (1997), 1121-1132. 


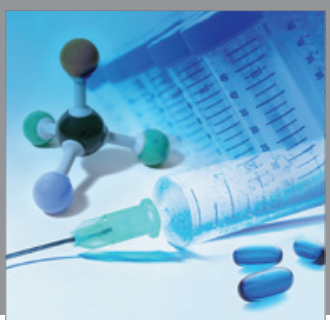

International Journal of

Medicinal Chemistry

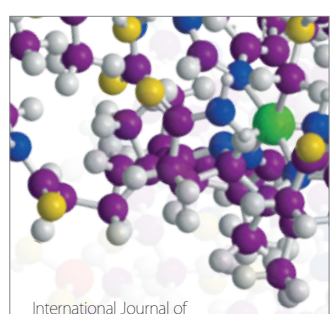

Carbohydrate Chemistry

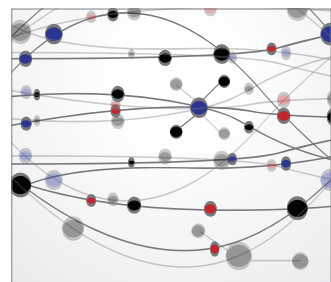

The Scientific World Journal
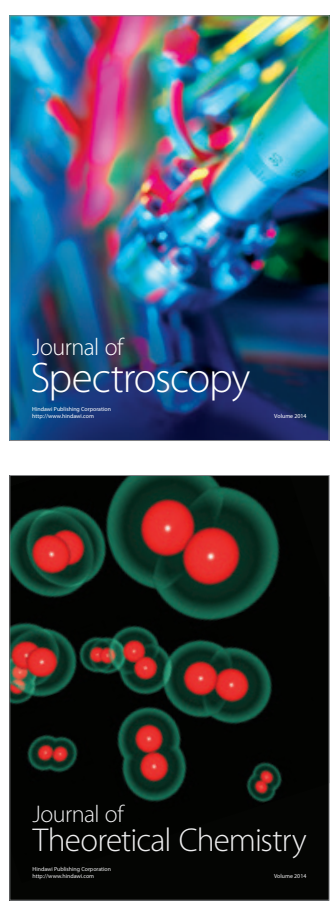
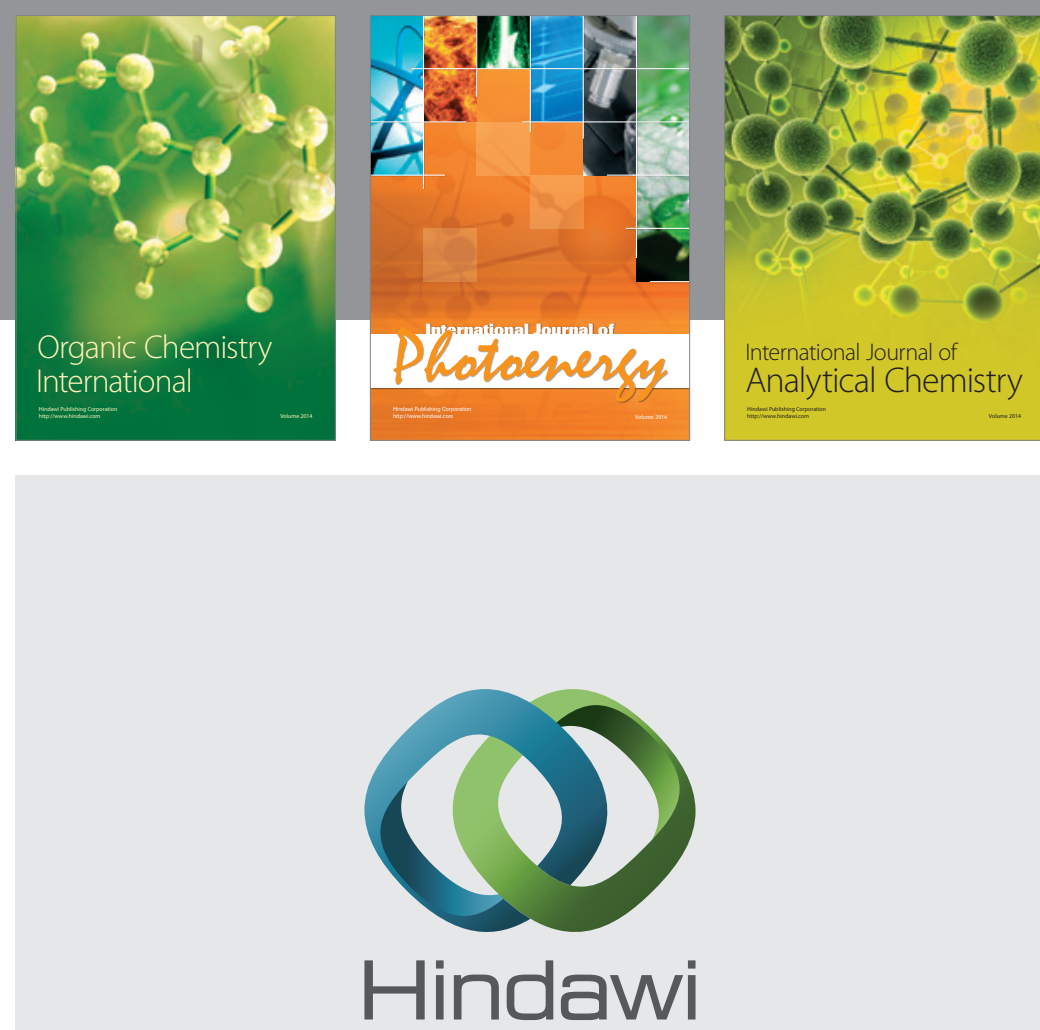

Submit your manuscripts at

http://www.hindawi.com
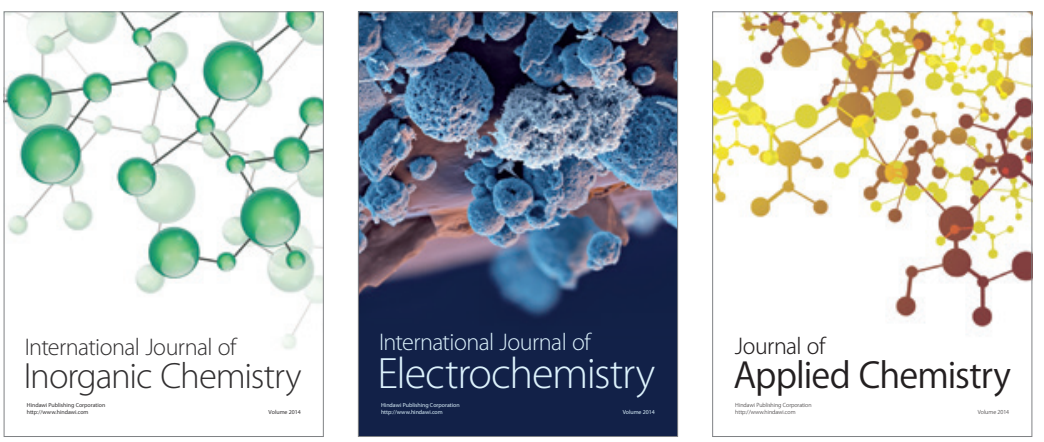

Journal of

Applied Chemistry
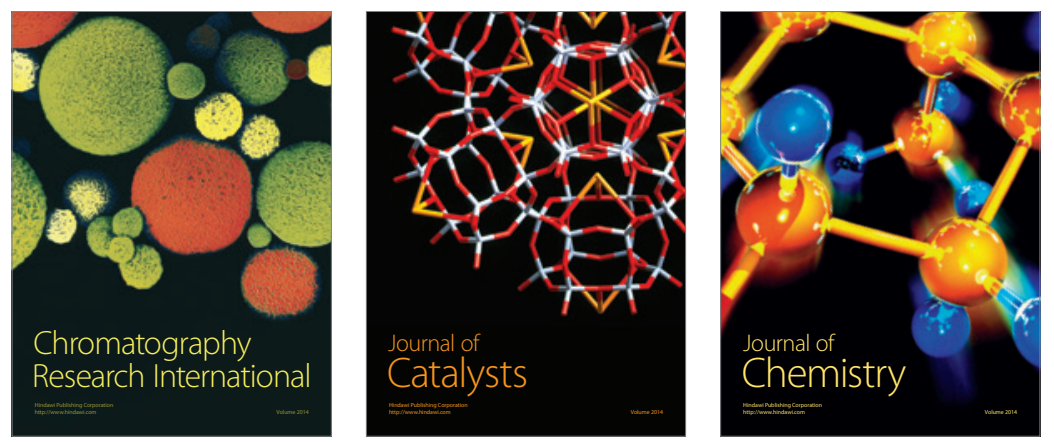
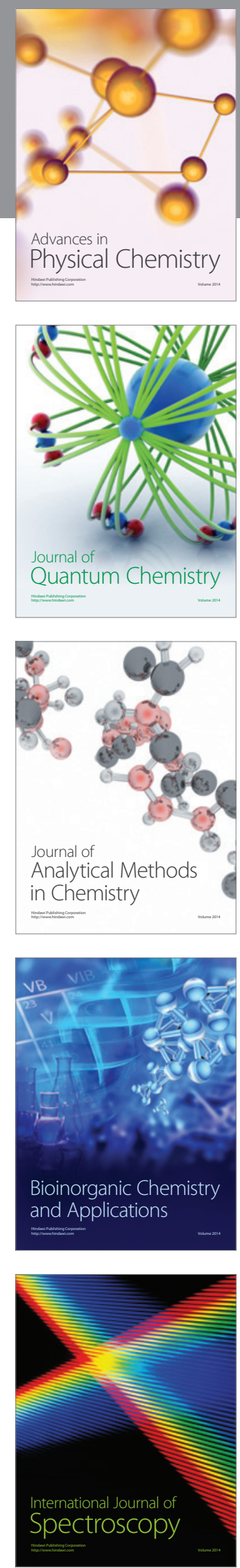